\title{
Rare Case of Retroperitoneal Tumor with Unusual Evolution
}

\author{
Lucian Sorin Andrei ${ }^{1}$, Adriana Corina Andrei ${ }^{2}$, Carmen-Monica Preda ${ }^{2}$, Cristina Dima ${ }^{1}$, \\ Larisa Elena Fulger ${ }^{2}$, Ioana Lupescü ${ }^{3}$, Vlad Herlea ${ }^{4}$
}

Corresponding author: Lucian Sorin Andrei, MD General Surgery and Hepatic Transplantation Department, Fundeni Clinical Institute, Bucharest

E-mail: sandrei741@yahoo.com
${ }^{1}$ General Surgery and Hepatic Transplantation Department, Fundeni Clinical Institute, Bucharest ${ }^{2}$ Gastroenterology and Hepatology Department, Fundeni Clinical Institute, Bucharest ${ }^{3}$ Radiology and Medical Imaging Department, Fundeni Clinical Institute, Bucharest ${ }^{4}$ Pathology Department, Fundeni Clinical Institute, Bucharest

\begin{abstract}
The retroperitoneum can host a wide spectrum of pathologies, including a variety of rare benign tumors and malignant neoplasms that can be either primary or metastatic lesions, whose correct diagnosis can be a challenge for the medical team and whose approach is often complex. We present the case of a patient diagnosed in June 2011, as a result of non-specific abdominal symptoms, with a small retroperitoneal mass with cystic appearance, whose affiliation could not be established. The evolution of this tumor was stable during the 3.5 years of clinical, biological and imaging follow-up, until January 2015 when the patient experienced neurological symptoms of lumbar nerve root compression which could have been associated with the increase in size of the retroperitoneal tumor. The therapeutic management was surgical tumor resection, and histopathology was suggestive for a pseudo-cyst, whose etiology remained unspecified. The peculiarity of the case is represented by the long silent evolution of a retroperitoneal mass, in which the association of neurological symptoms have posed problems of differential diagnosis, and in which the tumors etiology and sudden growth in size haven't been elucidated by the histopathological examination, where no cellular structures able to guide the diagnosis were detected. The curative treatment in these cases remains complete surgical resection of the tumor.
\end{abstract}

Key words: retroperitoneal tumor, CT scan, surgical resection

\section{INTRODUCTION}

The retroperitoneum can host a wide spectrum of pathologies, including a variety of rare benign tumors and malignant neoplasms that can be either primary or metastatic lesions $(1,2)$. Retroperitoneal tumors can cause a diagnostic dilemma and present several therapeutic challenges because of their rarity, relative late presentation and anatomical location, often in close relationship with several vital structures in the retroperitoneal space (1,3-5). 


\section{CASE REPORT}

We present the case of a non-smoker and nondrinker 36 years old male patient, who was admitted in June 2011 in the Gastroenterology Department presenting epigastric pain, abdominal bloating and intermittent loose stools, symptoms that started 2 months before admission.

His medical history includes: an acute entero-colitis (March 2011) and internal hemorrhoids, which were recently treated with rubber band ligation.

The clinical examination showed a patient with good general condition and normal weight, presenting a painful swelling of approximately $3 \mathrm{~cm}$ in diameter with a firm consistency on palpation of the epigastrium. No other clinical abnormalities were found.

All the laboratory findings were within normal limits except the cholesterol level which was elevated $(282$ $\mathrm{mg} / \mathrm{dl}$ ): amylase $=66 \mathrm{U} / \mathrm{l} ;$ AST $=29 \mathrm{U} / \mathrm{l}$; total bilirubin = $0,56 \mathrm{~g} / \mathrm{dl} ; \mathrm{GGT}=43 \mathrm{U} / \mathrm{l} ;$ no anemia;no inflammatory syndrome; the tumor markers tested were also within normal limits and viral hepatitis markers were negative. IgG antibodies for Echinococcus granulosus infection were also negative.

The abdominal ultrasound performed showed a mild liver steatosis and a well defined, 48/25 mm size, transonic mass situated in the retroperitoneal space which presented thin walls and no Doppler signal, whose affiliation could not be established. (fig. 1) Otherwise, there weren't other pathological abdominal findings.

The investigations continued with an abdominal CT scan with intravenous contrast which revealed two accumulations of fluid density, homogeneous, bounded by thin walls, located in the retroperitoneal space, one between the aorta and the cava vein $(35 / 30 \mathrm{~mm})$ and the other para-aortic $(27 / 22 \mathrm{~mm})$; some small lumbaraortic adenopathy (max. 12/6 mm); without any other modification. (fig. 2)

At that point, the stage diagnosis was of two retroperitoneal cystic masses in the middle lumbaraortic floor. The patient received symptomatic treatment and was called for reevaluation after 3 months. After this period, the symptoms improved, the abdominal ultrasound showed the same aspect and size of the retroperitoneal cystic mass and the tumoral markers remained negative. The patient was evaluated after another 3 months and then after another 6 months, without finding any modification.

In June 2013 the patient received an abdominal ultrasound in another medical unit which showed in the aortic-cave space a well-defined round mass, with-

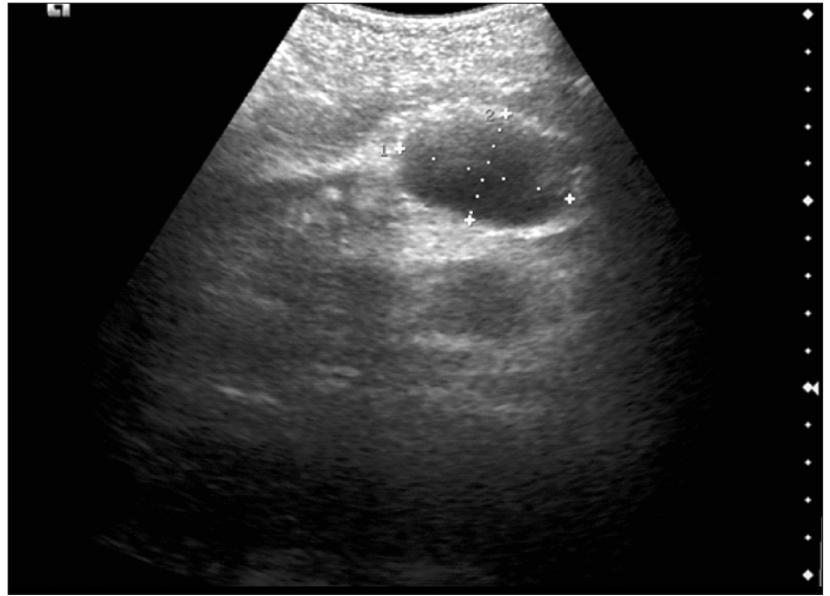

Figure 1 - Abdominal ultrasound examination: transonic retroperitoneal mass

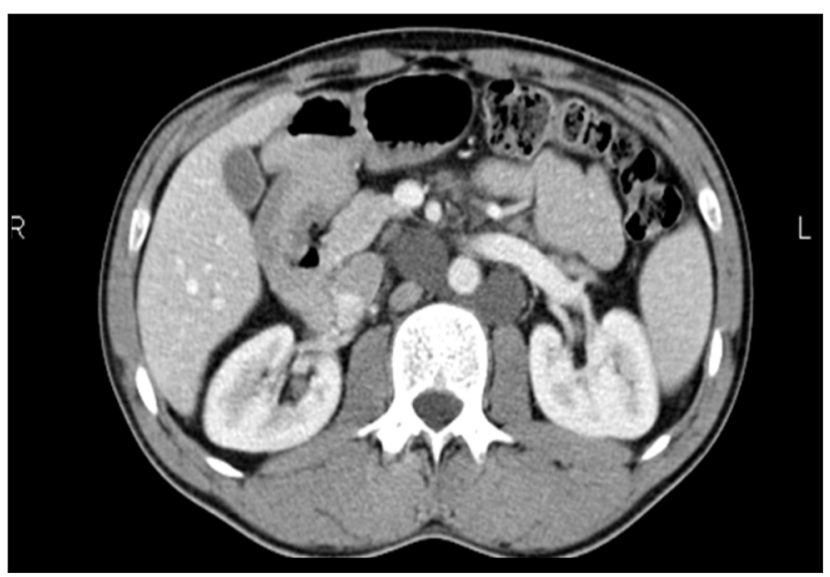

Figure 2 - Abdominal CT scan: retroperitoneal accumulations of fluid density

out Doppler signal, with transonic content, of 52/27 $\mathrm{mm}$ diameter and adjacent, a small adenopathy at $8 / 9$ $\mathrm{mm}$; no other modification was present.

In January 2015 the patient presented at the emergency room with lumbar pain and numbness in legs (in the left leg more than in the right). The spinal MRI showed lumbar scoliosis, median posterior protrusion of $L 4$ and left para-median protrusion of $L 5$ which marked the dural sac and a bilateral para-aortic fluid accumulation, at L2, with $38 \mathrm{~mm}$ and $26 \mathrm{~mm}$ diameter. The neurosurgeon concluded that the symptoms are not related to the spinal pathological findings of the MRI, which are not severe, and the patient does not have an indication for neurosurgical intervention, and recommended an abdominal CT scan with intravenous contrast to evaluate the retroperitoneal mass which appeared to be in contact with the lumbar spine at level L2. The CT scan (fig. $3 a, b$ ) revealed the increasing size of the cystic retroperitoneal mass: from $34 / 27 \mathrm{~mm}$ to $40 / 38 \mathrm{~mm}$ for the inter-aortic-cave cyst, 


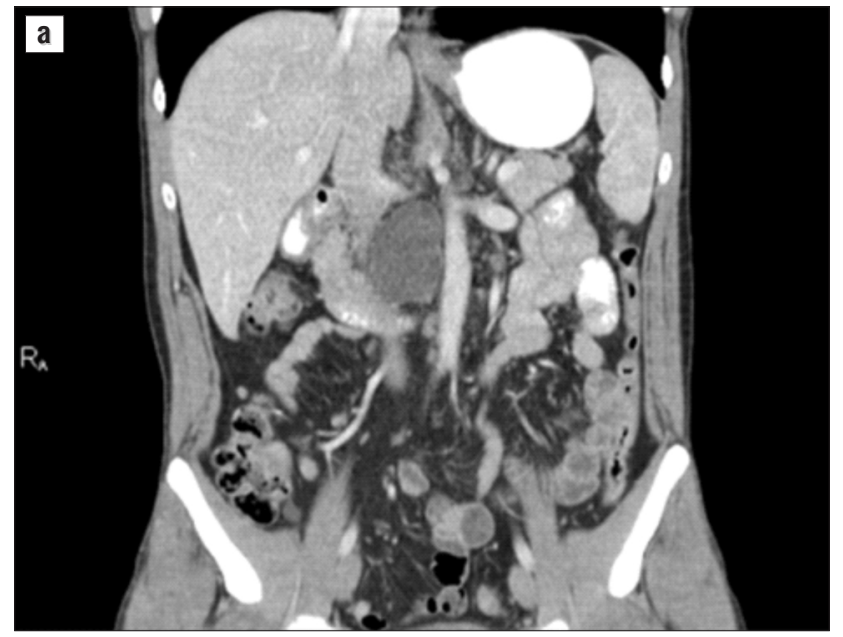

along with a stationary aspect of the left lumbar-aortic cyst $(25 \mathrm{~mm})$ - the two lesions seemed to communicate one with the other at the L2 level. There was an unchanged dimensional aspect of the lumbar-aortic adenopathy and no other changes.

In the context of the onset of abdominal pain, the patient was examined in a multidisciplinary committee in which a gastroenterologist and a radiologist joined the general surgeon, and a pre-operatory diagnose was established: retroperitoneal cystic tumor and abdominal lymphadenopathy. The committee decided to perform surgery with the main goal of tumor resection and lymph node biopsy, without other additional imaging procedures such as PET-CT, lymphoscintigraphy or lymphangiography that may have helped to establish an etiologic diagnosis, because of the accentuation of painful symptoms.

On February 2015 the patient was admitted in the surgery department andoperated by median laparotomy. The intraoperative aspect was: an hour glass shape cystic mass developed between the aorta and the cava vein, above the right renal pedicle,

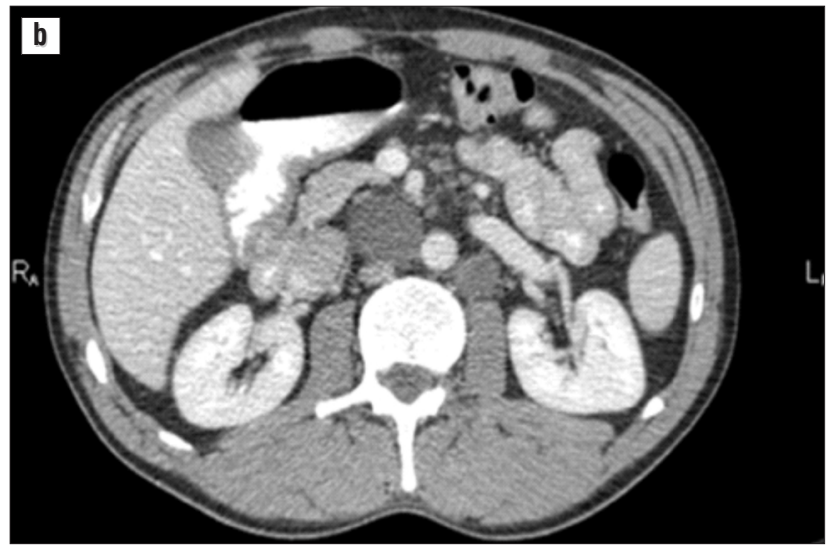

Figure $3 \mathrm{a}, \mathrm{b}$ - Retroperitoneal mass in contact with the lumbar (L2) vertebra

posterior of the duodenal-pancreatic space, extended para-aortal to the left. The right part of this mass measured about $4 \mathrm{~cm}$ and the left one about $5 \mathrm{~cm}$. It was impossible to establish during the surgical intervention any evidence of organ affiliation for this tumoral mass. None of the regional or lumbar-aortic lymph nodes showed intraoperative pathological aspect. The retroperitoneal tumor ablation en bloc with a satellite adenopathywas performed. When the tumor was dissected we discovered that it contained a yellowish substance with soft consistency. (fig. $4 a, b$ ) The pathology examination revealed connective tissue with mixed inflammatory infiltrate, macrophages with foamy cytoplasm, bordering hematic tissue and an immune-reactive nodule. (fig. 5) The aspect was suggestive for a retroperitoneal pseudo-cyst, but the absence of any cellular structure made it impossible to determine the origin of this tumor. The post-operative evolution was uncomplicated and the patient was discharged surgically cured, without any neurological symptoms.
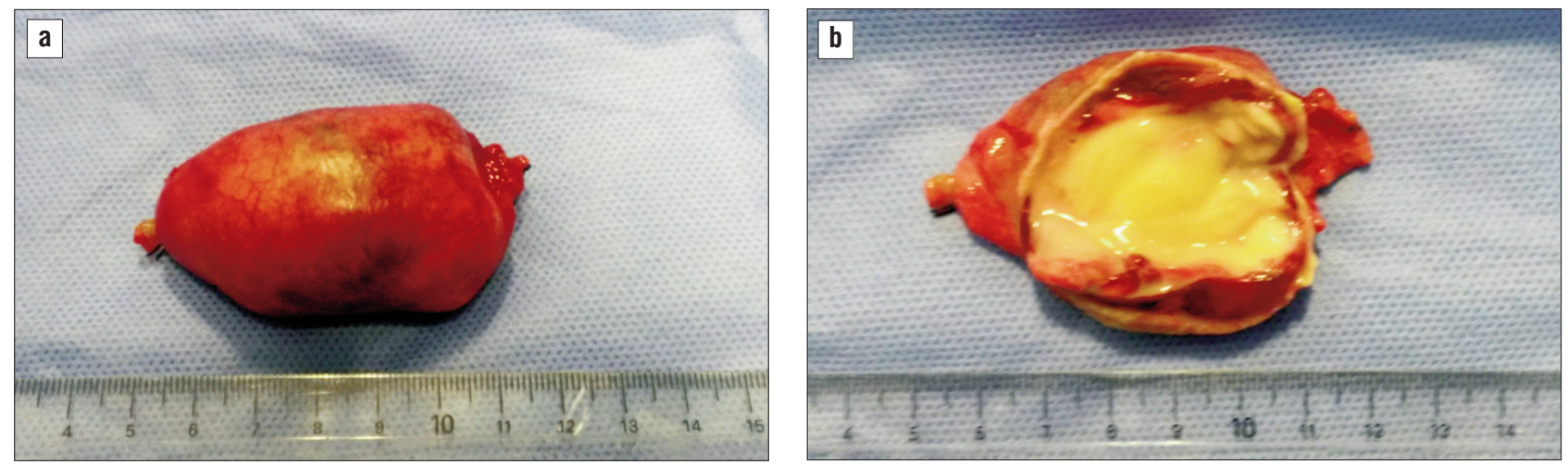

Figure 4 a, b - Retroperitoneal mass - surgically resected piece (macroscopic aspect) 


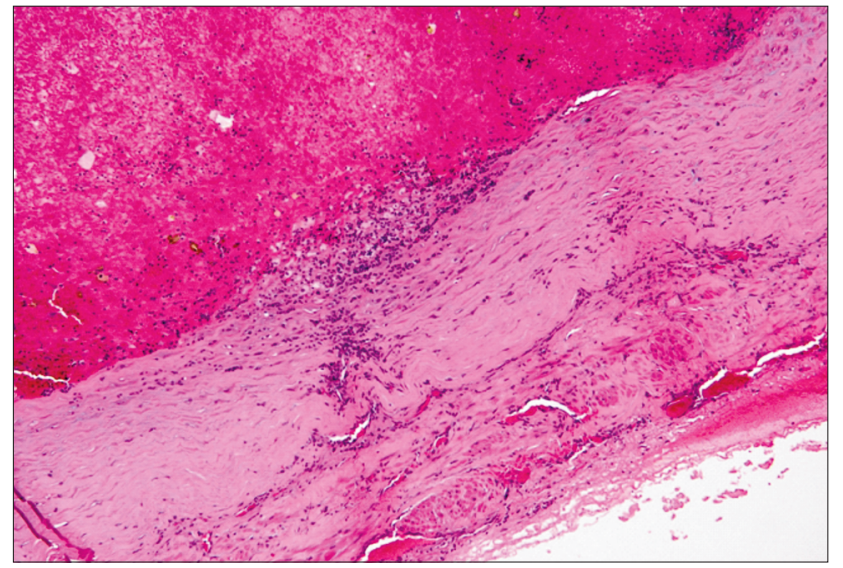

Figure 5 - Histological aspect (HE x 100) of the retroperitoneal pseudo-cyst

\section{DISCUSSIONS}

Primary retroperitoneal tumors represent a variety of lesions, with different treatments and prognoses (211). Sarcomas comprise a third of the retroperitoneal tumors, with two histological subtypes predominating: liposarcoma (70\%) and leiomyosarcoma (15\%) (1-3,12-14). Other retroperitoneal neoplasms include primary lymphoproliferative tumors (Hodgkin's and non-Hodgkin lymphoma) and epithelial tumors (renal, adrenal, pancreatic) or might represent metastatic disease from known or unknown primary sites (germ cell tumors, carcinomas, melanomas). Benign tumors can cause concern and are often an incidental finding during an investigation for unrelated symptoms. They may be referred to the surgeon under the suspicion of being a sarcoma $(15,16)$. The most common benign pathologies encountered in the retroperitoneum include benign neurogenic tumors (schwannomas, neurofibromas), paragangliomas (functional or nonfunctional), fibromatosis, renal angiomyolipomas and benign retroperitoneal lipomas (1,7-9).

Due to the inaccessibility of the retroperitoneal space and since these tumors often give no or nonspecific symptoms until they have reached a substantial size, they are usually large at presentation $(2,11,17)$. The clinical manifestations of retroperitoneal tumors are polymorphous and unspecific as they reveal themselves after a long latency period, in which the tumor had to develop enough to cause significant effects depending on its location and volume by compression, displacement, deformation or invasion of neighboring structures $(11-13,18)$. In our case, the retroperitoneal tumor determined first an infrequent mild epigastric pain and much later was associated with neurological symptoms which could have been related to an increase in the tumor size.

The imaging characteristics of the tumor usually do not allow, except for a few number of cases, conclusions regarding its benign or malignant nature, $(19,20)$ and only few data obtained by palpation are suggestive for malignancy when present (hardness, fixity). In general, as the tumor gets more stable and cannot be mobilized, the possibility of it being malignant increases $(5,11,12)$.

In our case, the echography was the one that highlighted the tumor and specified its cystic nature, but it must be underlined that abdominal ultrasound can not always specify the origin of the tumor from a specific organ, the vascular and visceral relations or possible invasion $(1,21)$.

The essential data provided by the computed tomography were represented by the highlighting of the retroperitoneal tumor which did not belong to a specified organ, the tumor size and its specific imagistic features, and especially the clarification of the relationships with adjacent structures $(7,10)$. In patients presenting with a retroperitoneal tumor, where the radiological appearance is uncertain or when the radiological appearance suggests a pathology where neo-adjuvant treatment may be appropriate as induction therapy (e.g. gastrointestinal stromal tumor, Ewing's sarcoma, teratoma), a pre-operative biopsy is mandatory. In this case, we were unable to perform a core needle biopsy due to the location (near the aorta, cava vein and right renal vein) and to the cystic type of the tumor $(4,5)$.

Complete surgical resection is the only potential curative treatment modality for the retroperitoneal tumors $(6,8,9)$. Resection of adjacent involved organs is frequently required and rates of resection of adjacent viscera are reported in large series from 34\% to $93 \%$. (10) The most common organs requiring resection are the colon, kidney, pancreas and spleen $(8-10,13)$.

The idea of a complex surgical team including a general surgeon and a neurosurgeon was first taken under consideration, but then abandoned because of the previous neurosurgical opinion and also based on the revaluation of the case by the expert committee, both considering that a team of general surgeons is the only one needed for this case.

The appropriate surgical approach was also discussed with the experts. The minimally invasive surgical approach would have been useful in evaluating lesions or for biopsy (by laparoscopy) or in the resection of the tumor (approachable through robotic surgery) especially for standardized locations, but was not considered appropriate for this situation because of the 
hourglass shape of the tumor which exerted mass effect on adjacent vascular structures. Close contact with important blood vessels in frequent in this tumors localization (22). In our case, the surgical team had chosen the median laparotomy approach and was able to realize the complete resection of the tumor, without damaging the vital blood vessels (aorta, cava vein and right renal vein) the tumor came in contact with.

The histology revealed a pseudo-cystic lesion of unknown origin, containing necrotic tissue - possible a completely necrotized tumor or lymphadenopathy, but the sudden increase in the size of the tumor after the 3.5 years follow up period remains unexplained in these conditions, in which no cellular structure has been identified. We can't exclude a retroperitoneal hematoma, but we couldn't identify a bleeding source or any cause for a retroperitoneal bleeding. We found only one relatively similar case in the medical literature which was reported in 2010 by Tomoyuki et al as an idiopathic retroperitoneal hematoma (23).

\section{CONCLUSIONS}

The retroperitoneum can host a wide spectrum of rare pathologies, including benign and malignant tumors. Patients usually present late because symptoms occur only when the tumor have reached a significant size. The diagnosis can be challenging in most of the cases, retroperitoneal tumors being best evaluated with good quality cross-sectional imaging. A complex, multidisciplinary medical team can establish the appropriate diagnostic and therapeutic strategy in this type of tumors. Complete surgical resection is the only potential curative treatment modality for retroperitoneal tumors. The histological examination can't properly diagnose some cases, such as ours, in which the main assumptions remain the retroperitoneal hematoma or a completely necrotized retroperitoneal tumor.

\section{REFERENCES}

1. Strauss DC, Hayes AJ, Thomas JM. Retroperitoneal tumours: review of management. Ann R Coll Surg Engl. 2011 May:93(4):275-80.

2. Van Roggen JF, Hogendoorn PC. Soft tissue tumours of the retroperi- toneum Sarcoma 2000:4(1-2):17-26.

3. Lehnert T, Cardona S, Hinz U, Willeke F, Mechtersheimer G, Treiber M, et al. Primary and locally recurrent retroperitoneal soft-tissue sarcoma: local control and survival. Eur J Surg Oncol. 2009 Sep;35(9):98693.

4. Strauss DC, Qureshi YA, Hayes AJ, Thway K, Fisher C, Thomas JM. The role of core needle biopsy in the diagnosis of suspected soft tissue tumours. J Surg Oncol. 2010 Oct 1;102(5):523-9.

5. Weiss SW, Goldblum JR. Enzinger and Weiss's Soft Tissue Tumors. $4^{\text {th }}$ edition. St Louis, Missouri, US: Mosby; 2001.

6. Strauss DC, Hayes AJ, Thway K, Moskovic EC, Fisher C, Thomas JM. Surgical management of primary retroperitoneal sarcoma. Br J Surg. 2010 May;97(5):698-706.

7. Clark MA, Fisher C, Judson I, Thomas JM. Soft-tissue sarcomas in adults. N Engl J Med. 2005 Aug 18;353(7):701-11.

8. Xu YH, Guo KJ, Guo RX, Ge CL, Tian YL, He SG. Surgical management of 143 patients with adult primary retroperitoneal tumor. World $J$ Gastroenterol. 2007 May 14:13(18):2619-21.

9. Hueman MT, Herman JM, Ahuja N. Management of retroperitoneal sarcomas. Surg Clin North Am. 2008 Jun;88(3):583-97.

10. Pisters PW. Resection of some - but not all - clinically uninvolved adjacent viscera as part of surgery for retroperitoneal soft tissue sarcomas. J Clin Oncol. 2009 Jan 1;27(1):6-8.

11. Gatita CE, Georgescu I, Nemes R. Difficulties in diagnosis of primitive retroperitoneal tumors. Current health sciences journal. 2010;36(3): 11-13.

12. Lucan M, Popescu I. Tratat de chirurgie - Bolile retroperitoneului, Volumul I "Urologie". București: Editura Academiei Romane; 2007. p 71-77.

13. Săvulescu F, Iordache I, Albița 0 , Hristea R, Dumitru C, lordache A, et al. Giant uterine leiomyoma. Chirurgia (Bucur). 2011 Sep-0ct;106(5): 665-8.

14. Setlacec D, Proca E, Popa C. Tumorile retroperitoneale primitive. București: Editura Medicala; 1986.

15. Venter A, Rosca E, Mutiu G, Daina L, Pirte A. Difficulties of diagnosis in retroperitoneal tumors. Rom J Morphol Embryol. 2013;54(2): 451-6.

16. Yamini Chitra V, Paramesh KN, Rithin Punjala Sai. How Common are Benign Retroperitoneal Tumors? A Case Series with Review of Literature. World Journal of Surgical Medical and Radiation Oncology. 2015;4:1.

17. Vasile I, Vîlcea D, Nemes $R$, Curcă $T$, Pasalega $M$, Calotă $F$, et al. Primary retroperitoneal tumors--diagnostic and therapeutic difficulties. Chirurgia (Bucur). 2005 Jan-Feb;100(1):27-33. Romanian

18. Popescu I, Georgescu S, Piertrareanu D, Herlea V, Popescu C. Vasculo-uretheral problems in the treatment of primitive retroperitoneal tumors. Chirurgia (Bucur). 1999 Jan-Feb;94(1):27-36.

19. Acar T, Harman M, Guneyli S, Gemici K, Efe D, Guler I, et al. Crosssectional Imaging Features of Primary Retroperitoneal Tumors and Their Subsequent Treatment. J Clin Imaging Sci. 2015 Apr 30;5:24

20. Fritchie KJ. Diagnostically Challenging "Fatty" Retroperitoneal Tumors. Surg Pathol Clin. 2015 Sep;8(3):375-97.

21. Urban ML, Palmisano A, Nicastro M, Corradi D, Buzio C,Vaglio A. Idiopathic and secondary forms of retroperitoneal fibrosis: A diagnostic approach. Rev Med Interne. 2015 Jan;36(1):15-21.

22. Qin Y, Yang Y, Yang H, Li J. Curved incision under the costal margin in retroperitoneal tumor surgery. Mol Clin Oncol. 2015 Jul:3(4):789-792.

23. Tomoyuki A, Masanori K, Osamu M, Takashi N. Idiopathic Retroperitoneal Hematoma. Case Rep Gastroenterol. 2010 Sep-Dec; 4(3): 318-322. 